\title{
A CIRCLE OF MODULAR GROUPS IN PU(2,1)
}

\author{
E. Falbel and P.-V. KoselefF
}

\begin{abstract}
We prove that there exists a circle of discrete and faithful embeddings of the triangle group of type $(2,3, \infty)$ in the automorphisms group of complex hyperbolic space. The proof is obtained by a construction of a fundamental domain using $\mathbf{C}$-spheres.
\end{abstract}

\section{Introduction}

Let $\Gamma$ be the triangle group of type $(p, q, \infty)$, that is, the abstract group presented by

$$
<\iota_{0}, \iota_{1}, \iota_{2}: \iota_{0}^{2}=1, \iota_{1}^{2}=1, \iota_{2}^{2}=1,\left(\iota_{0} \circ \iota_{1}\right)^{p}=1,\left(\iota_{0} \circ \iota_{2}\right)^{q}=1>.
$$

By an embedding of $\Gamma$ in $\mathbf{P} \widehat{\mathbf{U}(2,1)}$ (containing the holomorphic and the antiholomorphic transformations), the isometry group of the two dimensional complex ball (with the natural metric invariant under biholomorphisms), we will consider an homomorphism such that $\iota_{i}$ are mapped to anti-holomorphic generators and such that $\iota_{1} \circ \iota_{2}$ is parabolic. In this paper we prove:

Theorem 1.1. There exists a circle of discrete faithful embeddings of the triangle group $(2,3, \infty)$ in $\mathbf{P} \widehat{\mathbf{U}(2,1)}$. Up to conjugation in $\widehat{\mathbf{P U}(2,1)}$ the family is reduced to a quotient by the dihedral group $\mathbf{Z}_{\mathbf{2}} \times \mathbf{Z}_{\mathbf{2}}$. Moreover the family contains embeddings fixing a complex geodesic and embeddings fixing a totally real totally geodesic plane.

Embeddings of triangle groups of type $(p, q, \infty)$ in the neighborhood of an embedding fixing a complex geodesic were analyzed previously in [FK2]. A family connecting embeddings preserving a real and a complex geodesic for the triangle modular group was also obtained in [GuP1] independently, but there, fundamental domains were constructed for a subgroup of the triangle group. We prove discreteness of the embeddings by constructing explicitly fundamental domains using $\mathbf{C}$-spheres (see [FZ, FK1]).

The technique used in this paper is also sufficient to prove the same result for triangle groups $(p, q, \infty)$ for $p \leq q \leq 4$. In the case $3 \leq p, q$ we obtain an open neighborhood of a circle in the set of embeddings in $\mathbf{P} \widehat{\mathbf{U}(2,1)}$. On the other hand, for other triangle groups the technique proves the existence of

Received August 24, 2001. 
deformations of embeddings fixing either a complex geodesic or a totally real one.

\section{Complex hyperbolic space and its boundary}

In this section and the following we collect general results about complex hyperbolic space. As references, we use [G2], [FK1] and [FK2].

In dimension one, the disc and the half-plane are related by a Cayley transform. In dimension two, complex hyperbolic space

$$
H_{\mathbf{C}}^{2}=\left\{w \in \mathbf{C}^{2}:\left|w_{1}\right|^{2}+\left|w_{2}\right|^{2}<1\right\}
$$

is biholomorphic to

$$
V=\left\{z \in \mathbf{C}^{2}: \operatorname{Im}\left(z_{2}\right)>\left|z_{1}\right|^{2}\right\}
$$

using the Cayley transform

$$
C:\left(w_{1}, w_{2}\right) \mapsto\left(\frac{i w_{1}}{1+w_{2}}, i \frac{1-w_{2}}{1+w_{2}}\right) .
$$

The Heisenberg group $\mathbf{H}$ is the set of pairs $(z, t) \in \mathbf{C} \times \mathbf{R}$ with the product

$$
(z, t) \cdot\left(z^{\prime}, t^{\prime}\right)=\left(z+z^{\prime}, t+t^{\prime}+2 \operatorname{Im} z \bar{z}^{\prime}\right) .
$$

We identify the boundary of $V$ with the Heisenberg group via the map

$$
\left(z_{1}, z_{2}\right) \mapsto\left(z_{1}, \operatorname{Re}\left(z_{2}\right)\right)
$$

The distribution obtained by translating the $t=0$ plane at the origin is of contact type and makes the Heisenberg group a homogeneous contact manifold. Moreover, a homogeneous conformal structure on the distribution can be defined by translating the conformal class defined by the flat metric on the $t=0$ plane at the origin.

It is well known that the boundary $S^{3}$ of the complex hyperbolic space can be identified to the one point compactification $\overline{\mathbf{H}}$ of the Heisenberg group. The group of all conformal transformations of $\overline{\mathbf{H}}$ is isomorphic to $\mathbf{P} \widehat{\mathbf{U}(2,1)}$ (see [G2]).

There are two kinds of totally geodesic submanifolds of real dimension 2 in $H_{\mathbf{C}}^{2}$ : complex geodesics (represented by $H_{\mathbf{C}}^{1} \subset H_{\mathbf{C}}^{2}$ ) and totally real geodesic 2planes (represented by $H_{\mathbf{R}}^{2} \subset H_{\mathbf{C}}^{2}$ ). Each of these totally geodesic submanifolds is a model of the hyperbolic plane.

We define $\mathbf{C}$-circles to be the intersections of $S^{3}$ with the boundaries of totally geodesic complex submanifolds $H_{\mathbf{C}}^{1}$ in $H_{\mathbf{C}}^{2}$. Analogously, we define $\mathbf{R}$ circles to be the intersections of $S^{3}$ with the boundaries of totally geodesic totally real submanifolds $H_{\mathbf{R}}^{2}$ in $H_{\mathbf{C}}^{2}$. 
Definition 2.1. The inversion on an $\mathbf{R}$-circle is the non-trivial element of $\mathbf{P} \widehat{\mathbf{U}(2,1)}$ that fixes it point-wise.

There is, then, a one-to-one correspondence between inversions and $\mathbf{R}$-circles. For instance the transformation $\hat{m}(z, t)=(\bar{z},-t)$ on the Heisenberg group is the inversion that fixes point-wise the $\mathbf{R}$-circle $\operatorname{Im}(z)=0$.

We will also consider points to be $\mathbf{C}$-circles and refer to them as degenerate $\mathbf{C}$ circles. Any two points (possibly coincident) in the Heisenberg group determine a unique $\mathbf{C}$-circle (possibly degenerate) containing them (see [G2]). One can easily show that the $\mathbf{C}$-circles that are invariant under a given inversion are precisely those that intersect twice the associated $\mathbf{R}$-circle. As a permutation of two points gives the same $\mathbf{C}$-circle, the set of invariant $\mathbf{C}$-circles is the Möbius band $S^{1} \times S^{1} / \mathbf{Z}_{2}$.

Given an $\mathbf{R}$-circle, a union of invariant $\mathbf{C}$-circles that is homeomorphic to a sphere is called a $\mathbf{C}$-sphere (see [FZ]). Fundamental domains in this paper will be bounded by (pieces of) $\mathbf{C}$-spheres.

2.1. R-circles and C-circles in the Heisenberg model. We denote by $I_{0}$ the inversion on $\mathbf{R}_{0}$ (see [G2] and [FK1] for details)

$$
\mathbf{R}_{0}: r^{2}+i z=-e^{-2 i \theta} \text {. }
$$

$\mathbf{R}_{0}$ is given in cylindrical coordinates by $r=\sqrt{-\cos (2 \theta)}, z=\sin (2 \theta)$. The following lemma describes in cylindrical coordinates the homogeneous contact distribution in the Heisenberg group.

Lemma 2.2. The contact plane at $M=(r, \theta, z)$ is : $z-Z-2 r(\cos (\theta) Y-$ $\sin (\theta) X)=0$.

Any infinite $\mathbf{R}$-circle lies in the contact plane at each of its points.

In the Heisenberg model, C-circles are either vertical lines or else ellipses that belong to the contact plane of their centers and whose projections onto the $z$-plane are circles.

The Möbius band of all invariant $\mathbf{C}$-circles described in the previous section can be parametrized by the surface defined by all their centers. In order to obtain that surface we need to impose that the $\mathbf{C}$-circle having center $(X, Y, Z)$ and radius $R$ intersects twice the $\mathbf{R}$-circle $\mathbf{R}_{0}$. After some calculations, one obtains:

Lemma 2.3 (see [FK1]). Invariant $\mathbf{C}$-circles under the inversion on $\mathbf{R}_{0}$ are centered in the surface of centers $\{z=\sin (2 \theta)\}$ and have radius $R^{2}=r^{2}+$ $\cos (2 \theta)$. If $r^{2}+\cos (2 \theta) \leq 0$ we say that the $\mathbf{C}$-circle is imaginary.

As a $\mathbf{C}$-sphere around $\mathbf{R}_{0}$ is union of invariant $\mathbf{C}$-circles, one can describe it by giving a path (called an axis) in the surface of centers. But the condition that it is homeomorphic to a sphere imposes certain restrictions to that path. One of the simplest sufficient conditions is to impose that the $\mathbf{C}$-circles in the family defined by the axis be non-intersecting and non-linked pairwise (see section 3.1). 
Definition 2.4. $\mathbf{R}_{r, \theta, \alpha}$ is the infinite $\mathbf{R}$-circle passing through $M=(r, \theta, \sin (2 \theta))$ whose projection on the $z$-plane is the line of slope $\tan (\alpha)$.

Observe that $\mathbf{R}_{r, \theta, \alpha}$ is horizontal if and only if $\alpha=\theta \bmod \pi$.
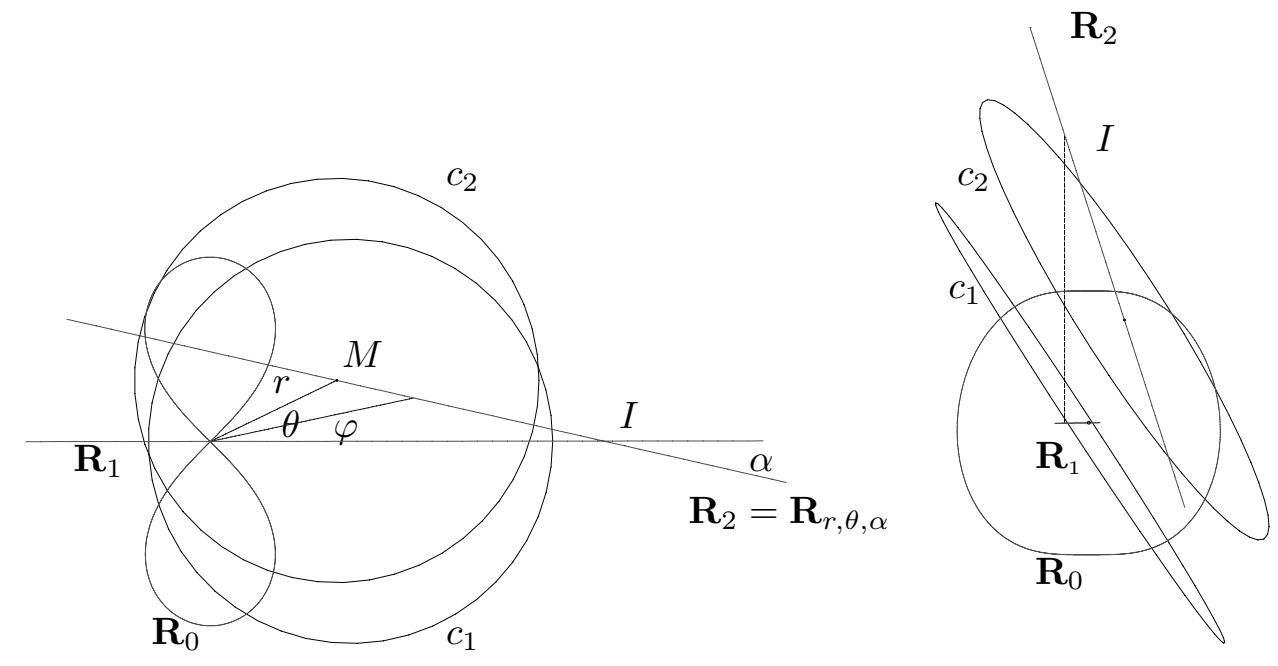

Left: Projection on the $z$-plane of the configuration of $\mathbf{R}_{0}, \mathbf{R}_{1}$ and $\mathbf{R}_{2}(\theta)$ for the modular group showing the invariant $\mathbf{C}$-circles $c_{1}$ and $c_{2}$. In the picture we fixed $\theta=\pi / 7 . \mathbf{R}_{2}(\theta)$ has polar coordinates $\rho$ and $\varphi$.

Right: A different projection of the configuration.

2.2. Configurations. Let $\iota_{0}, \iota_{1}$ and $\iota_{2}$ be the standard generators of the triangle group $(2,3, \infty)$. Consider an embedding $r:(2,3, \infty) \rightarrow \widehat{\mathbf{P}} \widehat{\mathbf{U}(2,1)}$ such that $r\left(\iota_{i}\right)=I_{i}$ are inversions fixing three $\mathbf{R}$-circles $\mathbf{R}_{i}$ and such that $I_{1} \circ I_{2}$ is parabolic.

Observe that $I_{1} \circ I_{0}$ is an elliptic element of order 2 and $I_{2} \circ I_{0}$ is an elliptic element of order 3. $I_{1} \circ I_{0}$ is conjugate to an element in $U(2)$. Its eigenvalues may be \pm 1 . Similarly $I_{2} \circ I_{0}$ could have eigenvalues $1, e^{2 \pi / 3}, e^{-2 \pi / 3}$. There are 6 possible families of embeddings.

As R-circles are tangent to the contact distribution, one can define an angle $\theta$ between them whenever they intersect. In that case, the eigenvalues of the composition of inversions will be 1 and $2 \theta$. If they do not intersect but are linked one can turn one of them by an angle $\varphi$ around an invariant $\mathbf{C}$-circle under both inversions until they intersect defining a new angle $\theta$. In that case, 
the eigenvalues of the composition will be $2 \varphi$ and $2 \theta$ (see [FZ] for details). In order to parameterize the configurations one needs to impose for each pair of $\mathbf{R}$-circles a fixed pair of angles.

In [FK2], the family of embeddings such that $I_{1} \circ I_{0}$ have both eigenvalues \pm 1 and such that $I_{2} \circ I_{0}$ have both eigenvalues $e^{2 \pi / 3}, e^{-2 \pi / 3}$ are called of type $(\pi / 2,-\pi / 2, \pi / 3,-\pi / 3)$. It contains two distinguished discrete and faithful representations, namely one preserving a complex line and one preserving a real line. A parameterization of those embeddings is given by the following proposition.

Consider in the Heisenberg group $\mathbf{R}_{0}$ to be the standard $\mathbf{R}$-circle, $\mathbf{R}_{1}$ to be the infinite $\mathbf{R}$-circle given by the $x$-axis and $\mathbf{R}_{2}$ to be the family of infinite $\mathbf{R}$-circles $\mathbf{R}_{2}(\theta)=\mathbf{R}_{r, \theta, \alpha}$ where

$$
\alpha=-\pi / 2+3 \theta, r^{2}=-\frac{\cos (2 \theta-\pi / 3) \cos (2 \theta+\pi / 3)}{\cos (2 \theta)}=-\frac{\cos (6 \theta)}{4 \cos ^{2}(2 \theta)}
$$

for $\frac{\pi}{12} \leq \theta \leq \frac{\pi}{6}$. We denote by $I_{0}$ the inversion fixing $\mathbf{R}_{0}$, by $I_{1}$ the inversion fixing the $\mathbf{R}_{1}$ and by $I_{2}(\theta)$ the inversion on the $\mathbf{R}$-circle $\mathbf{R}_{2}(\theta)$.

Theorem 2.5 (FK2). The representations $r$ of the triangle group $(2,3, \infty)$ with $r\left(\iota_{0}\right)=I_{0}, r\left(\iota_{1}\right)=I_{1}$ and $r\left(\iota_{2}\right)=I_{2}(\theta)$ form a connected component of the representation space up to conjugations in $\mathbf{P} \widehat{\mathbf{U}(2,1)}$. Moreover if $\theta=\pi / 12$ the representation fixes a complex line (a $\mathbf{C}$-fuchsian representation) and if $\theta=$ $\pi / 6$, the representation fixes a totally real totally geodesic plane ( $a \mathbf{R}$-fuchsian representation).

A circle of representations in $\widehat{\mathbf{P U}(2,1)}$ is obtained by conjugating the group by the inversions fixing the $\mathrm{x}$-axis and the $\mathrm{y}$-axis. The index two subgroup generated by the order two and order three elements will have, a fortiori, a circle of representations in $\mathbf{P} \mathbf{U}(2,1)$. The space of representations modulo conjugation in $\mathbf{P U}(2,1)$ is obtained by the quotient of this circle by a $\mathbf{Z}_{2}$-action (a rotation by $\pi)$.

\section{Families of non-linked C-circles}

3.1. Non-linking condition. The linking condition can be formulated as follows.

Let $C_{1}$ and $C_{2}$ be two $\mathbf{C}$-circles and $M_{1}, M_{2}$ be their centers with parameters $\left(r_{1}, \theta_{1}, z_{1}\right)$ and $\left(r_{2}, \theta_{2}, z_{2}\right)$. Their radius are $R_{i}$. They are not linked if and only if

$$
E=d^{4}-2\left(R_{1}^{2}+R_{2}^{2}\right) d^{2}+\left(R_{1}^{2}-R_{2}^{2}\right)^{2}+(h+4 S)^{2}>0,
$$

where $h=z_{2}-z_{1}$ and $S=1 / 2\left(x_{1} y_{2}-x_{2} y_{1}\right), d^{2}=\left(x_{1}-x_{2}\right)^{2}+\left(y_{1}-y_{2}\right)^{2}$ (see [FK1]). 
3.2. Good axis in the surface of centers. Let $M(\theta)$ be lying on the surface of centers of $\mathbf{R}_{0}$, that is $M(\theta)=(r, \theta, \sin (2 \theta))$. It defines an invariant $\mathbf{C}$-circle $C(\theta)$ for $\mathbf{R}_{0}$ with radius $R^{2}(\theta)=r^{2}(\theta)+\cos (2 \theta)$.

Lemma 3.1. Let $r_{0} \leq 1$. The curve $M(\theta)=\left(r_{0}, \theta, \sin (2 \theta)\right)$ in the surface of centers defines a family of invariant non-linked $\mathbf{C}$-circles for $\mathbf{R}_{0}$, when $\theta \in$ $[-\pi / 2, \pi / 2]$.

Proof. Let $r_{1}=r_{2}=r_{0}$, we get $1 / 4 E$

$$
\begin{aligned}
& =-r_{0}^{2}\left(\cos \left(2 \theta_{1}\right)+\cos \left(2 \theta_{2}\right)-2 \cos \left(\theta_{1}+\theta_{2}\right)\right)+\sin \left(\theta_{1}-\theta_{2}\right)^{2} \\
& =\left(1-\cos \left(\theta_{1}-\theta_{2}\right)\right)\left(\left(\cos \left(\theta_{1}-\theta_{2}\right)+r_{0}^{2} \cos \left(\theta_{1}+\theta_{2}\right)\right)+\left(r_{0}^{2} \cos \left(\theta_{1}+\theta_{2}\right)+1\right)\right) \\
& =\left(1-\cos \left(\theta_{1}-\theta_{2}\right)\right)\left(\cos \left(\theta_{1}\right) \cos \left(\theta_{2}\right)\left(1+2 r_{0}^{2}\right)+\sin \left(\theta_{1}\right) \sin \left(\theta_{2}\right)\left(1-2 r_{0}^{2}\right)+1\right) .
\end{aligned}
$$

But

$$
\cos \left(\theta_{1}\right) \cos \left(\theta_{2}\right)\left(1+2 r_{0}^{2}\right)+\sin \left(\theta_{1}\right) \sin \left(\theta_{2}\right)\left(1-2 r_{0}^{2}\right)+1 \geq \min \left(2 r_{0}^{2}, 2-2 r_{0}^{2}\right) \geq 0 .
$$

We call such a curve a good axis (cf. [FK1]).

3.3. Dilated C-circles. We construct a two parameter family of unlinked Ccircles using dilations $l_{\lambda}:(z, t) \mapsto\left(\lambda z, \lambda^{2} t\right)$ in the Heisenberg group.

Lemma 3.2. Let $r(\theta), \theta \in I$ be a curve in the surface of centers such that corresponding $\mathbf{C}$-circles are not linked (good axis in [FK1]). Let $C_{\lambda}(\theta)=l_{\lambda}(C(\theta)$ ) be the two-parameter family of dilated $\mathbf{C}$-circles $C(\theta)$ by dilation $l_{\lambda}$. For any $\lambda_{1}, \lambda_{2}>0$ and $\left(\theta_{1}, \theta_{2}\right) \in I \times I$. If $r\left(\theta_{1}\right) r\left(\theta_{2}\right) \cos \left(\theta_{1}+\theta_{2}\right) \geq-1$, then $C_{\lambda_{1}}\left(\theta_{1}\right)$ and $C_{\lambda_{2}}\left(\theta_{2}\right)$ are not linked.

Remark 3.3. Observe that the surfaces $C_{\lambda}(\theta)$ (constant $\lambda$ ) are disjoint. Moreover, by the previous lemma, as $r(\theta)=r_{0} \leq 1$ is a good axis, the conclusion of the lemma is true in that case.

Proof. Let $C(\theta)$ be a $\mathbf{C}$-circle of center $M(\theta)$ and radius $R(\theta)$, then $C_{\lambda}(\theta)$ has center $M_{\lambda}(\theta)$ and radius $\lambda R$. Linking formula for $C_{\lambda_{1}}\left(\theta_{1}\right)$ and $C_{\lambda_{2}}\left(\theta_{2}\right)$ is

$$
\begin{aligned}
F= & \lambda_{1}^{4}+\lambda_{2}^{4}-2 \lambda_{1}^{2} \lambda_{2}^{2}\left(2 r_{2}^{2} \cos \left(2 \theta_{1}\right)+2 r_{1}^{2} \cos \left(2 \theta_{2}\right)+\cos \left(2 \theta_{1}-2 \theta_{2}\right)\right) \\
& +4 \lambda_{1} \lambda_{2} r_{1} r_{2}\left(\lambda_{1}^{2}+\lambda_{2}^{2}\right) \cos \left(\theta_{1}+\theta_{2}\right) \\
= & \lambda_{1}^{2} \lambda_{2}^{2} E+\left(\lambda_{1}-\lambda_{2}\right)^{2}\left(\lambda_{1}^{2}+\lambda_{2}^{2}+2 \lambda_{1} \lambda_{2}\left(1+2 r_{1} r_{2} \cos \left(\theta_{1}+\theta_{2}\right)\right)\right. \\
\geq & 0
\end{aligned}
$$

when $E \geq 0$ and $r_{1} r_{2} \cos \left(\theta_{1}+\theta_{2}\right) \geq-1$. 


\subsection{Invariant $\mathrm{C}$-circles for an infinite $\mathrm{R}$-circle.}

Lemma 3.4. Let $C(\varphi), \varphi_{1} \leq \varphi \leq \varphi_{2}$ be a family of $\mathbf{C}$-circle of radius $R(\varphi)$ centered at $M(\varphi)$ which projects to a line in the the xy-plane. Suppose that $\varphi \mapsto R(\varphi)$ is differentiable and let $d(\varphi)$ be the horizontal distance between $M(\varphi)$ and $M\left(\varphi_{2}\right)$. If $\left[\frac{\mathrm{d} R^{2}(\varphi)}{\mathrm{d} \varphi}\right]^{2}>4 R(\varphi)^{2}\left[\frac{\mathrm{d} d(\varphi)}{\mathrm{d} \varphi}\right]^{2}$, then the family $C(\varphi)$ has no links.

Proof. In this case $R(\varphi)-d(\varphi)$ is monotonic and circles are not linked because their projections are not.

\section{Construction of a fundamental domain}

Theorem 4.1. The embeddings of the triangle group defined by $\left\langle I_{0}, I_{1}, I_{2}(\theta)\right\rangle$ are faithful and discrete for all $\pi / 12 \leq \theta \leq \pi / 6$.

It follows from the following construction.

Theorem 4.2. For each $\pi / 12 \leq \theta \leq \pi / 6$, there exits three surfaces $\mathbf{S}_{0}, \mathbf{S}_{1}$ and $\mathbf{S}_{2}$ such that

- each $\mathbf{S}_{i}$ is foliated by invariant $\mathbf{C}$-circles under $I_{i}$,

- $c_{1}=\mathbf{S}_{0} \cap \mathbf{S}_{1}$ is an invariant $\mathbf{C}$-circle under both $I_{0}$ and $I_{1}$,

- $c_{2}=\mathbf{S}_{0} \cap \mathbf{S}_{2}$ is an invariant $\mathbf{C}$-circle under both $I_{0}$ and $I_{2}(\theta)$,

- $\mathbf{S}_{1} \cap \mathbf{S}_{2}$ is the point at infinity.

Remark 4.3. Observe that $\mathbf{S}_{0}$ is homeomorphic to an annulus. On the other hand, $\mathbf{S}_{1}$ and $\mathbf{S}_{2}$ are homeomorphic to discs. The fundamental domain is homeomorphic to the solid of revolution obtained from the classical fundamental domain for the triangle modular group when it is revolved around the $x$-axis.

Remark 4.4. Those surfaces (pieces of $\mathbf{C}$-spheres) are called $\mathbf{C}$-surfaces in [FZ, FK1]. An application of Poincaré's theorem for complex hyperbolic geometry [FZ, FK2] states then that the region bounded by the three surfaces in the Heisenberg group is a fundamental domain for the triangle group. In [FK2] we proved the theorem for $\theta$ in a neighborhood of $\pi / 12$.

Proof. We are going to construct 3 surfaces, $\mathbf{S}_{0}, \mathbf{S}_{1}$, and $\mathbf{S}_{2}$.

- $\mathbf{S}_{0}$ is an annulus,

- $\mathbf{S}_{1}$ is a disk,

- $\mathbf{S}_{2}$ is a disk.

For the sake of exposition we're going to break $\mathbf{S}_{1}$ into a near part, $N \mathbf{S}_{1}$, and a far part $F \mathbf{S}_{1}$. The near part of $\mathbf{S}_{1}$ will intersect $\mathbf{S}_{0}$ in a circle. The far part of $\mathbf{S}_{1}$ is unbounded. We break up $\mathbf{S}_{2}$ in the same way.

To construct $\mathbf{S}_{0}$, we need to choose a curve in the surface of centers of $\mathbf{R}_{0}$. One endpoint of this curve is determined. It must be the circle $c_{2}$, which is fixed by both $I_{0}$ and $I_{2}$. Note that the other end of the curve is not determined because $\mathbf{R}_{1}$ lies in the surface of centers of $\mathbf{R}_{0}$. Given that one endpoint of our 
curve is fixed, the simplest thing to do it is to take an arc of the circle, having this endpoint, and then lift it to the surface of centers. This is the curve - call it $M(\psi)$ - which detemines $\mathbf{S}_{0}$. Here $\psi \in[0, \theta]$.

We would like to make $F \mathbf{S}_{1}$ and $F \mathbf{S}_{2}$ as simple as possible. So, let's make them each contained in a plane, and also make them translates of each other. So, $F \mathbf{S}_{1}$ and $F \mathbf{S}_{2}$ will be contained in parallel planes. The plane containing $F \mathbf{S}_{1}$ must contain $\mathbf{R}_{1}$ and also must be parallel to $\mathbf{R}_{2}$. There is a unique vertical line $V$ which intersects both $\mathbf{R}_{1}$ and $\mathbf{R}_{2}$, and any contact plane centered on $V$ has the desired property. Thus, we make $F \mathbf{S}_{1}$ by taking expanding concentric circles, about the point $V$ intersect $\mathbf{R}_{1}$. All these circles lie in the desired plane. To get $F \mathbf{S}_{2}$ we translate $F \mathbf{S}_{1}$ upward until it contains points on $\mathbf{R}_{2}$.

So far we have not quite pinned down $F \mathbf{S}_{1}$ because we haven't specified the smallest circle in it. Note that $C_{0}(0)$ is centered on $\mathbf{R}_{1}$. Some dilation of $C_{0}(0)$ is centered at the point $V$ intersect $\mathbf{R}_{1}$. We take the smallest circle in $F \mathbf{S}_{1}$ to be this dilation of $C_{0}(0)$. Once again, we take $F \mathbf{S}_{2}$ to be the upward translation of $F \mathbf{S}_{1}$.

For $N \mathbf{S}_{1}$ note that we already have the two ending $\mathbf{C}$-circles. One of them is $C_{0}(0)$ and the other one is a certain dilation of $C_{0}(0)$. To get the family of unlinked $\mathbf{C}$-circles interpolating these two $\mathbf{C}$-circles, we take the union of the dilations of $C_{0}(0)$ up to the one centered at $V$ intersected with $\mathbf{R}_{1}$.

For $N \mathbf{S}_{2}$ note that we already have the two ending $\mathbf{C}$-circles. The small one is $C_{0}(\theta)$. The big one is the translation taking $V \cap \mathbf{R} 1$ to $V \cap \mathbf{R}_{2}$ of the last dilated $C_{0}(0)$ centered in $V \cap \mathbf{R}_{1}$. That is, the big $\mathbf{C}$-circle is a translation of a dilation of $C_{0}(0)$. Note that the small one is a translation of a dilation of $C_{0}(\theta)$, where the translation and dilation are trivial. Let's examine the nontrivial translation+dilation more carefully, with a view towards interpolation between the trivial and the nontrivial. $C_{0}(0)$ is dilated until its center lies exactly below $\mathbf{R}_{2}$, and then tanslated up until it lies on $\mathbf{R}_{2}$. For interpolation, take any $\varphi \in[0, \theta]$. Dilate $C_{0}(\varphi)$ until its center lies below $\mathbf{R}_{2}$ and then translate until the center lies on $\mathbf{R}_{2}$. The union of all these circles is $N \mathbf{S}_{2}$.

\section{- Technical steps -}

For each fixed $\pi / 12 \leq \theta \leq \pi / 6$, the $\mathbf{R}$-circle $\mathbf{R}_{2}(\theta)$ is parametrized by an angle $\varphi$ such that $\alpha<\varphi<\alpha+\pi$, where $\alpha=-\pi / 2+3 \theta$. We have $[\rho(\varphi) \cos (\varphi), \rho(\varphi) \sin (\varphi), Z(\varphi)]$ with

$$
\rho(\varphi)=r(\theta) \sin (\alpha-\theta) / \sin (\alpha-\varphi)=\frac{\sqrt{-\cos (6 \theta)}}{2 \cos (3 \theta-\varphi)}
$$


and

$$
\begin{aligned}
Z(\varphi) & =\sin (2 \theta)+2 r(\theta) \rho(\varphi) \sin (\theta-\varphi) \\
& =\sin (2 \theta)+\frac{\cos 6 \theta \sin (\theta-\varphi)}{2 \cos 2 \theta \cos (3 \theta-\varphi)} \\
& =\frac{\sin (3 \theta+\varphi)}{2 \cos (3 \theta-\varphi)}
\end{aligned}
$$

$\mathbf{R}_{2}(\theta)$ intersects the surface of centers of $\mathbf{R}_{0}$ for $\varphi=\theta$, and

$$
\rho^{2}(\theta)=r^{2}(\theta)=-\frac{\cos (6 \theta)}{4 \cos ^{2}(2 \theta)} .
$$

This intersection point determines a C-circle $c_{2}$ which should be $\mathbf{S}_{0} \cap \mathbf{S}_{2}$.

On the other hand any point in the $x$-axis is the center of an invariant circle under both $I_{0}$ and $I_{1}$. For instance, the origin is the center of the equator. To construct $\mathbf{S}_{0}$ we must choose one of those invariant $\mathbf{C}$-circles.

$$
-\mathbf{S}_{0}-
$$

For each fixed $\pi / 12 \leq \theta<\pi / 6$, we choose the family of invariant $\mathbf{C}$-circles under $I_{0}$ to be the family $C_{0}(\psi), 0 \leq \psi \leq \theta$, defined by the arc in the surface of centers

$$
M(\psi)=(r(\theta), \psi, \sin (2 \psi))
$$

of constant radius. The endpoint of this arc is the invariant $\mathbf{C}$-circle $c_{1}$ under both $I_{0}$ and $I_{1}$. Its center is $(r(\theta), 0,0)$ in the Heisenberg group. By lemma 3.1 this arc defines the surface $\mathbf{S}_{0}$.

$$
-\mathbf{S}_{1}-
$$

The $\mathbf{C}$-circle $c_{1}=C_{0}(0)$ will be dilated into the $\mathbf{C}$-circles $C_{1}(t)=l_{t}\left(c_{1}\right)$ (where $l_{t}$ is Heisenberg dilation by $t$ ) whose centers belong to $\mathbf{R}_{1}$, and $1 \leq t \leq \frac{\rho(0)}{\rho(\theta)}$. By lemma 3.2 the dilated circles are disjoint and unlinked. Their union does not intersect $\mathbf{S}_{0}$, and will be part of the surface $\mathbf{S}_{1}$. We complete the surface $\mathbf{S}_{1}$ by a union of concentric $\mathbf{C}$-circles centered at the point $\rho(0)$ of the $x$-axis with increasing radii up to infinity: this is $F \mathbf{S}_{1}$. We have $c_{1}=\mathbf{S}_{0} \cap \mathbf{S}_{1}$.

$$
-\mathbf{S}_{2}-
$$

Each C-circle $C_{0}(\varphi)$, for $0 \leq \varphi \leq \theta$, will be dilated into a $\mathbf{C}$-circle $C_{2}^{\prime}(\varphi)=$ $l_{\frac{\rho(\varphi)}{\rho(\theta)}} C_{0}(\varphi)$ (where $l_{\frac{\rho(\varphi)}{\rho(\theta)}}$ is Heisenberg dilation by $\left.\frac{\rho(\varphi)}{\rho(\theta)}\right)$ whose center belongs to the curve

$$
\mathbf{R}_{2}^{\prime}=\left[\rho(\varphi) \cos (\varphi), \rho(\varphi) \sin (\varphi),\left(\frac{\rho(\varphi)}{\rho(\theta)}\right)^{2} \sin (2 \varphi)\right] .
$$

$\mathbf{R}_{2}^{\prime}$ and $\mathbf{R}_{2}$ have same projection onto the $x y$-plane. But the height of $\mathbf{R}_{2}^{\prime}$ is

$$
Z^{\prime}(\varphi)=\left(\frac{\rho(\varphi)}{\rho(\theta)}\right)^{2} \sin (2 \varphi)=\frac{\cos ^{2} 2 \theta \sin 2 \varphi}{\cos ^{2}(3 \theta-\varphi)}
$$


We call $\mathbf{S}_{2}{ }^{\prime}$ the surface determined by those $\mathbf{C}$-circles.

We translate (by a vertical translation on the Heisenberg group) each $\mathbf{C}$-circle $C_{2}^{\prime}(\varphi)$ in such a way that its center belongs to $\mathbf{R}_{2}$. We thus obtain a family $\mathbf{S}_{2}$ of invariant $\mathbf{C}$-circles $C_{2}(\varphi)$ for $\mathbf{R}_{2}$, for $0 \leq \varphi \leq \theta$. We complete the family by the union of concentric $\mathbf{C}$-circles centered at the point of $\mathbf{R}_{2}$ that projects over the $x$-axis: this is $F \mathbf{S}_{2}$.

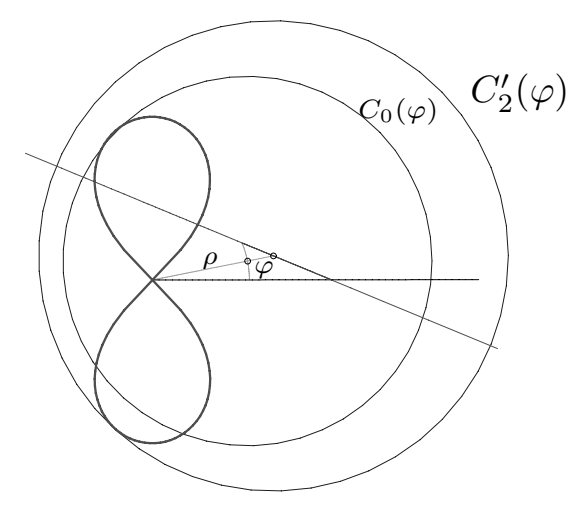

Left: Projection on the $z$-plane of the C-circles of $C_{0}(\varphi)$ and $C_{2}^{\prime}(\varphi)$; the second one is obtained from the first one by a dilation. In this configuration $\theta$ was fixed.

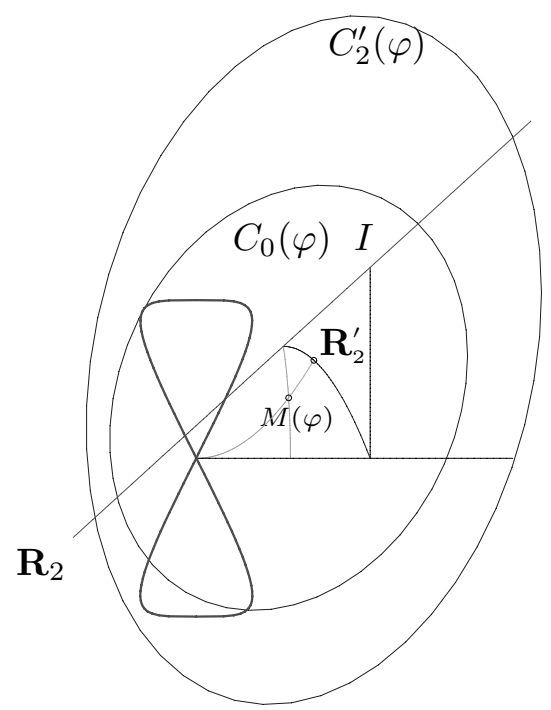

Right: A space view of the configuration. We see the curve $\mathbf{R}_{2}^{\prime}(\varphi)$, which is a $l_{\frac{\rho(\varphi)}{\rho(\theta)}}(M(\varphi))$ where $M(\varphi)$ is defined in equation 1 . Shown in the picture, the effect of the dilation at angle $\varphi$.

$\mathbf{S}_{0}, \mathbf{S}_{1}$ and $\mathbf{S}_{2}{ }^{\prime}$ have no linked $\mathbf{C}$-circles. Any two of the $\mathbf{C}$-circles of $\mathbf{S}_{0}, \mathbf{S}_{1}$ or $\mathbf{S}_{2}{ }^{\prime}$ are not linked because they are all obtained by dilation of $\mathbf{C}$-circles of $\mathbf{S}_{0}$ (by lemma 3.2).

$\mathbf{S}_{2}$ has no linked C-circles. We will apply lemma 3.4 to the family of $\mathbf{C}$-circles $C_{2}(\varphi)$. They all have centers in $\mathbf{R}_{2}$. Observe that $\mathbf{R}_{2}^{\prime}$ and $\mathbf{R}_{2}$ project onto the same line in the $x y$-plane, so it is sufficient to show that the projections of the family $C_{2}^{\prime}(\varphi)$ are disjoint. Let $\rho_{0}=\rho(\theta)$, we have that $C_{2}^{\prime}(\varphi)=l_{\frac{\rho(\varphi)}{\rho_{0}}} C_{0}(\varphi)$ has 
radii given by $R^{2}(\varphi)=\left(\frac{\rho(\varphi)}{\rho_{0}}\right)^{2}\left(\rho_{0}^{2}+\cos (2 \varphi)\right)$. Using lemma 3.4, we compute

$$
\begin{gathered}
{\left[\frac{\mathrm{d} R^{2}(\varphi)}{\mathrm{d} \varphi}\right]^{2}-4 R(\varphi)^{2}\left[\frac{\mathrm{d} d(\varphi)}{\mathrm{d} \varphi}\right]^{2}} \\
=4 \frac{\sin (\alpha-\theta)^{4}}{\sin (\varphi-\alpha)^{6}}\left(\cos (\varphi+\alpha)^{2}+\rho_{0}^{2} \cos (2 \alpha)-\rho_{0}^{4} \sin (\varphi-\alpha)^{2}\right)
\end{gathered}
$$

But

$$
\begin{aligned}
\cos (\varphi+\alpha)^{2}+\rho_{0}^{2} \cos (2 \alpha)-\rho_{0}^{4} \sin (\varphi-\alpha)^{2} & \geq \cos (\varphi+\alpha)^{2}+\rho_{0}^{2} \cos (2 \alpha) \\
& -\sin (\varphi-\alpha)^{2} \\
& =\cos (2 \alpha)\left(\rho_{0}^{2}+\cos (2 \varphi)\right) \\
\geq & 0
\end{aligned}
$$

because $\cos (2 \alpha)=\cos (6 \theta-\pi) \geq 0$ for $\frac{\pi}{12} \leq \theta \leq \frac{\pi}{6}$.

$\mathbf{R}_{2}^{\prime}$ is below $\mathbf{R}_{2}$. Let $Z^{\prime}(\varphi)$ and $Z(\varphi)$ be the height of the centers of $C_{2}^{\prime}(\varphi)$ and $C(\varphi)$. We compute

$$
\begin{aligned}
Z(\varphi)-Z^{\prime}(\varphi) & =\frac{\sin (3 \theta+\varphi)}{2 \cos (3 \theta-\varphi)}-\frac{\cos ^{2} 2 \theta \sin 2 \varphi}{\cos ^{2}(3 \theta-\varphi)} \\
& =\frac{1}{4 \cos ^{2}(3 \theta-\varphi)}\left(2 \sin (3 \theta+\varphi) \cos (3 \theta-\varphi)-4 \cos ^{2} 2 \theta \sin 2 \varphi\right) \\
& =\frac{1}{4 \cos ^{2}(3 \theta-\varphi)}\left(\sin 6 \theta+\sin 2 \varphi-4 \cos ^{2} 2 \theta \sin 2 \varphi\right) \\
& =\frac{1}{4 \cos ^{2}(3 \theta-\varphi)}\left(\sin 6 \theta-\frac{\sin 6 \theta}{\sin 2 \theta} \sin 2 \varphi\right) \\
& =\frac{\sin 6 \theta}{4 \sin 2 \theta \cos ^{2}(3 \theta-\varphi)}(\sin 2 \theta-\sin 2 \varphi)
\end{aligned}
$$

We thus deduce that $Z^{\prime}(\varphi) \leq Z(\varphi), 0 \leq \varphi \leq \theta$.

$\mathbf{S}_{0}, \mathbf{S}_{1}$ and $\mathbf{S}_{2}$ are disjoint. $\mathbf{S}_{0}, \mathbf{S}_{1}$ and $\mathbf{S}_{2}{ }^{\prime}$ are disjoint as seen previously. It remains to show that $\mathbf{S}_{2}$ is disjoint from the other surfaces. As $\mathbf{S}_{2}$ is above $S 2^{\prime}$, a fortiori $\mathbf{S}_{2}$ does not intersect $\mathbf{S}_{0}$ and $\mathbf{S}_{1}$.

\section{Acknowledgement}

We thank S. Markham and J. Parker for help with trigonometry, and R. Schwartz for improving the exposition. 

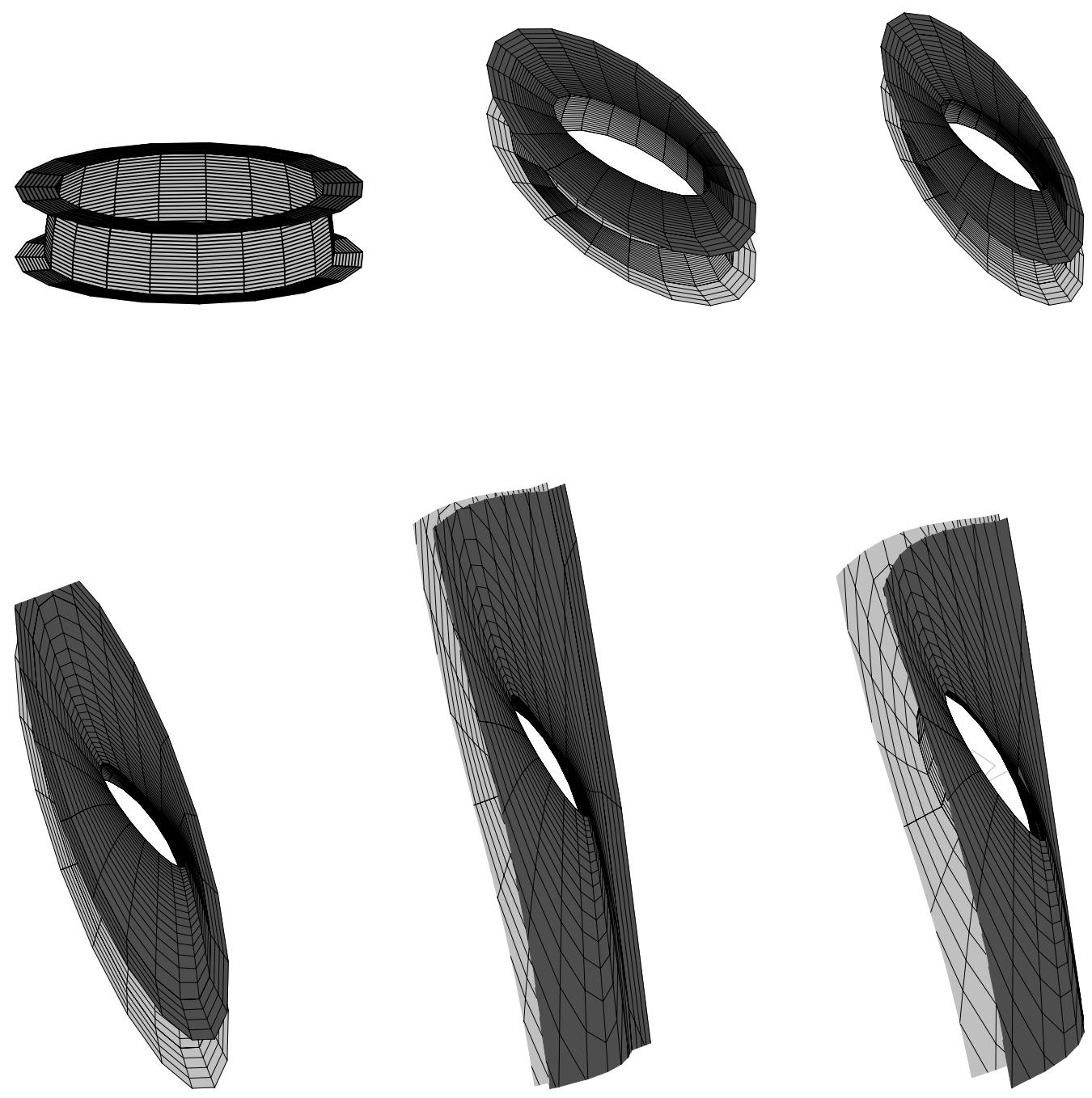

Six fundamental domains for $\theta$ between $\pi / 12$ and $\pi / 6$. Remark that scaling is not constant. The completion of the surfaces by concentric $\mathbf{C}$-circles is partially drawn. The first upper left image corresponds to the embedding fixing a complex geodesic, while the last one fixes a totally real geodesic.

\section{References}

[FK1] E. Falbel, P.-V. Koseleff, Flexibility of ideal triangle groups in complex hyperbolic geometry, Topology, 39 (2000), no. 6, 1209-1223. 
[FK2] _ Rigidity and Flexibility of triangle groups in complex hyperbolic geometry, Topology, 41 (2002), no. 4, 767-786.

[FZ] E. Falbel, V. Zocca, A Poincaré's polyhedron theorem for complex hyperbolic geometry, J. Reine Angew. Math. 516 (1999), 133-158.

[G2] W. Goldman, Complex hyperbolic geometry, Oxford Mathematical Monographs. Oxford Science Publications. The Clarendon Press, Oxford University Press, New York, 1999.

[GP] W. Goldman, J. Parker, Complex hyperbolic ideal triangle groups, J. Reine Angew. Math. 425 (1992), 71-86.

[GuP1] N. Gusevskii, J. Parker, Complex hyperbolic quasi-Fuchsian groups and Toledo's invariant, preprint, 2001.

Institut de Mathématiques, Université Pierre et Marie Curie, 4, Place Jussieu, F-75252 PARIS, France.

E-mail address: falbel@math.jussieu.fr

E-mail address: koseleff@math.jussieu.fr 